\title{
SEGMENTATION IN CHICK'S IMAGE USING ARTIFICIAL NEURAL NETWORK
}

\author{
Dr.A.SenthilRajan
}

\begin{abstract}
This work proposal is for localization of suspicious noise in chick's images by selecting regions of interest. The presence of salt and pepper noise, speckle noise in an image becomes a difficult task to delimit a lesion. Some techniques of digital processing were applied in 100 chick's images in order to minimize the noise allowing posterior extraction of the contour. The segmentation techniques used to extract the contour was performed by means of the artificial neural network selforganizing map. The various metric evaluation were used to compare the proximity of the automatically obtained area with the manual outline of a animal husbandry, resulting in percent values of $93 \%$ to accuracy, $68 \%$ to sensitivity and $98 \%$ to positive predictive value.
\end{abstract}

Keywords - Noise, Speckle, Extraction, Lesion.

\section{Introduction}

Mycoplasma gallisepticum (MG) is a bacteria-like organism that causes respiratory disease primarily in chickens and turkeys but it can also infect pigeons, ducks, and wild birds. MG infection in chickens is also known as Chronic Respiratory Disease (CRD, and chickens who are exposed to it will carry the disease for the rest of their lives even if (after treatment) they do not show signs of it anymore. Birds that have been exposed or recovered from MG should not be used as breeding stock. MG does not make people sick and eating eggs from infected birds will not hurt, but the production of chicks form farm will drastically reduce in weights as well in total number.

Aware of the new techniques in digital image processing which allow earlier diagnosis and treatment have been studied and implemented to increase the survival rate of the chicks. Currently the well experienced animal husbandry doctors can judge the type of disease easily. Therefore, the chances of a false positive result lead to unnecessary removal of chicks from the farm and this also leads to total reduction of money gain. Besides the risk of taking the individual chicks from the lot in the farm will take more time as well as the increase the labor cost. Thus it is necessary to investigate alternative methods for the detection of infected chicks.

Dr.A.Senthil Rajan , Director

Alagappa University

Karaikudi, INDIA
With recent technology advances, the image captured from any modern phones will be processed by sending the image to image analyzer or processed by any image processing software available on the phone. The technique involves removal of noise, segmentation and procedure to recover the chicks from infection. The detection of chicks can cause errors due to high subjectivity. Trying to minimize these errors and to help early detection of Chronic Respiratory Disease, computer aided detection schemes has been developed to improve diagnostic accuracy and support to minimize the errors while screening the image.

\section{Image Database}

For this research paper, ninety cases of chicken images were selected which contains suspicious mass of bulges, different types of noises in the image. The images are captured in Agni farm feeds in karuvelanpatti , Madurai , Tamilnadu , India. The chicks images were acquired by a VIVO V9 4 GB RAM | 64 GB ROM | Expandable Upto 256 GB, 6.3 inch Full HD+ Display, 16MP + 5MP Dual Rear Camera | 24MP Front Camera, 3260 mAh Li-ion Battery, Qualcomm Snapdragon 626 Processor. The captured image will be sent to the central server for processing.

For each chicks image was analyzed by the animal husbandry to determine the location of one or more suspicious bulges ,cut regions and different types of noises deemed necessary using the digital image processing software IDL ( Interactive data Language)

\section{Pre-processing Techniques}

To find the detection of bulges in an image, pre-processing techniques were applied in order to find the effective removal of various noises and also clear bulges in a chick's images. According to [8] the application of pre-processing improves a significantly segmentation instead of process it directly in the original images.

To reduce the speckle noise present in an image, before segmentation, the ROI were initially submitted to median filter [9] with neighborhood window size of $5 \times 5$, to minimize the additive noise. Then, the contrast has been increased by image equalization or histogram [14]. Finally, the noise can be 
removed and smoothing occurred in 8X8 neighborhood window.

One of the great difficulty in captured image is the image is corrupted due to noise and presents a low quality. Therefore, the segmentation which is the most important task for computer aided detection. This segmentation process attempts to identify objects with similar characteristics, separating unsuspicious regions of the background. Another task of this process is the obtained an accurate representation of boundaries, whose characteristics can be evaluated and used in classifying of the type of lesion.

Considering the above difficulties this research paper presents the evaluation of segmentation by artificial neural network and self-organizing map to automatic detection of any bulges contour in regions of interest from chicken images.

\section{A. Segmentation on Image}

Artificial neural network are intelligent models grounded in mathematical functions developed to resemble the workings of bulges in chicken, modeling the achievement of a particular task. Thus, the self-organizing map network, which does not have a fixed topology, being modified according to the problem and data distribution. It is composed of 20 neurons in its input layer, fully connected to the topologic square grid of size 10X10. The grid performs the calculation of mathematical functions and the necessary adjustments. These connections are associated with synaptic weights which stock the knowledge of the model serving as a mechanism of response to input received by each neuron

The self-organizing map network uses unsupervised learning, wherein only the pairs of input are supplied and there is not a desired output. It is characterized by learning through examples of the external environment, where the algorithm adapts the parameters for a given data set, seeking convergence to a solution,

The learning process occurs via the self-organizing of thee topological map in a competitive way. The input pattern creates a dispute among neurons to be activated. The activated neuron is called "winning unit" and their weights will be updated in the training, as well as the weight of their neighbors.

\section{Proposed Algorithm}

Training: A sample network is created in order to restrict the process and to connect it in only two classes (according to those presented in the sample).Then, the network receives the input image and classifies them according to the values obtained in the previous step.
Continuation: It returns the sampling until no longer observe any significant changes on map.

Sampling: Two classes of samples are collected (values corresponding to the white color set at 255 and black at 0 normally these values are assigned similar to the values assigned in Digital image processing) and, during training stage, the output data must converge.

Similarity and updating: Find the similar pattern (winner neuron). The convergence process should be varied out in a maximum of 1500 comparison. Then, the minimization of the distance may be calculated using the Euclidian distance. Finally the similar pattern image is found and its area of influence in relation to the neighborhood. Now update the similar pattern involved in this radius (copy of pixel values to the neighborhood pixels).

Region of Interest: The main purpose is to eliminate the remaining unwanted regions in image. Consider that ROI has only one lesion and this is the region that has the larger area. Thus, all connected components were found and the region of highest area were checked, which was considered as a lesion. Then, it was verified the connectivity, being removed all pixels not connected to this region. Finally, analyzed the occurrence of internal values, these pixels will be added to the segmented area, forming a single region.

\section{Evaluation of Results using AI}

Quantitative measurements were obtained in order to evaluate the accuracy of the segmentation technique. For this, animal husbandry traced manually the lesion contour. The denominated area given by the animal husbandry will be as "good truth" (GT), was compared with that obtained by the automatic segmentation method proposed. If the segmented region coincided with the GT, it was denoted as Truth Positive (TP), however if the region was considered negative by the observer, it was counted as a False Positive (FP). On the other hand, if the pixels not belong to the GT and were classified as such, were defined as a Truth Negative (TN), but if there were present in the segmentation, were considered as a False Positive (FP). From this, it can be derive three metrics: accuracy, sensitivity and positive predictive value (PPV)

The accuracy measures the number of correct classifications in relation to the total of classified elements, according

$$
\text { Accuracy }=(\mathrm{TP}+\mathrm{TN}) /(\mathrm{TP}+\mathrm{TN}+\mathrm{FP}+\mathrm{FN})
$$

The sensitivity represents the proportion of elements in the GT that were correctly identified. The calculation is performed from

$$
\mathrm{SEN}=\mathrm{TP} /(\mathrm{TP}+\mathrm{FN})
$$


The positive predictive value, finally, corresponds to the proportion of pixels correctly classified in the segmented region.

$$
\mathrm{PPV}=\mathrm{TP} /(\mathrm{TP}+\mathrm{FP})
$$

The processing of an ultrasound image is a challenge one because of due to various noise interference and imprecision of the boundaries. In order to minimize the noise problem, the filter techniques like median filter image histogram, image equalization is applied.

After minimizing the noise and increase the contrast between the background and the object, the segmentation technique using the artificial neural network type for self-organizing map for better to locate and mark the boundaries of lesion contour as accurately as possible. Trying to maintain just the suspicious mass on the segmented image, the image has to apply a post processing techniques to remove adjacent regions which not belong to the object of interest and junction of internal valleys. The last procedure is to obtain the contour overlapped on the input ROI for a qualitative visual evaluation, allowing verifying the consistence of the result presented by the proposed method and the lesion present in the image.

The result for segmentation the process of removing remaining spurious and the overlapping of contour can be shown in the below figure

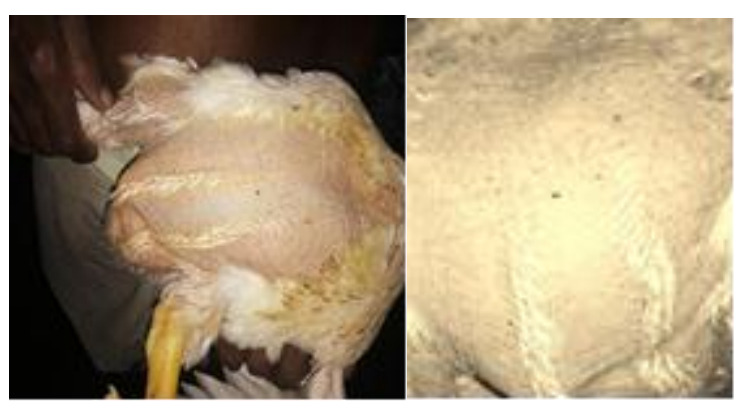

Figure1. Original Image and Region of Interest

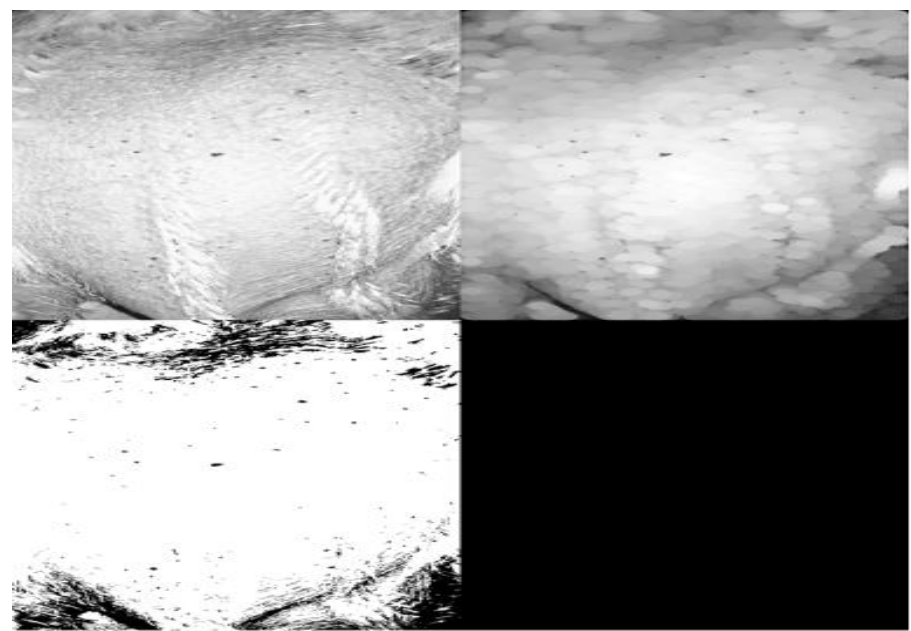

Figure 2. Top row: Original, After Filtering

Bottom row: After Threshold, More Threshold

Using the metrics the quantitative verification result can be obtained. This will be done by the subjectivity given by the visual process which is dependent of the observer. To make this is possible; an experience animal husbandry will observe the lesions manually in 100 chick's images, trying to achieve the highest accuracy as possible.

\section{vII. Conclusion}

The main idea of this paper is to evaluate the segmentation techniques capable of precisely designing the boundaries of lesions found on breast chicks images, automatically. However, this is a difficult task due to the presence of salt and pepper noise, which significantly degrades the image quality and difficult the discrimination of some details. The preprocessing techniques employed, in some cases, degraded the boundaries of the lesions, nevertheless allowed the enhancement of the contrast between the background and the object of interest, contributing to the subsequent detection.

The differential of the ANN to other process techniques is their learning ability making it an algorithm that enables the occurrence of variations in the boundaries of the lesion if the image is submitted to a new training process because the corresponding values for the weights are given randomly, what modifies the convergence of the data. This network has some algorithmic complexity and its computational cost increases with the image size, considering the training and classification time. However, as the use of region of interest the processing time is low.

The segmentation method proposed allows greater distinction between isolated noise and the object of interest. Thus the segmentation is more uniform, with accurate and smoother boundaries, besides to being not so susceptible to noise. 
Proc. of the Seventh Intl. Conf.on Advances in Computing, Communication and Information Technology - CCIT 2018 Copyright (C) Institute of Research Engineers and Doctors, USA. All rights reserved.

ISBN: 978-1-63248-162-7 DOI: 10.15224/978-1-63248-162-7-06

\section{References}

1. Bezdek.J.C, "Pattern Recognition with Fuzzy Objective Function Algorithms". New York: Plenum, 1981.

2. Bodin.F and Seznec.A, Skewed associativity improves program performance and enhances predictability. IEEE Transactions on Computers, 46(5):530-544, May 1997.

3. Chan.T and Wu.H.R, "Adaptive impulse detection using centerweighted median filters", IEEE Signal Processing Letters, vol. 8, no. 1, pp. 1-3, 2001.

4. Chu.C.K and Marron.J.S, "Choosing a kernel regression estimator (with discussion)," Statist. Sci., vol. 6, pp. 404-436, 1991.

5. Dhamaparikar.S, Krishnamurthy.P, Sproull.T.S, Lockwood.J.W, Deep packet Inspection Using Parallel Bloom Filters", IEEE Micro, vol.24, no. 1, pp.52-61, 2004.

6. Eggers.S, Emer.J, Levy.H,Levy.J, Lo.J, Stamm.R and Tullsen.D, "Eimultaneous Multithreading: A platform for Next generation Processors", IEEEMocri, vol.17, no.5, PP.12-19, 1997.

7. Dr.E.Ramaraj, A.Senthilrajan, "Parallel Sorting Algorithm", $12^{\text {th }}$ International workshop on Future Trends of Distributed Computing Systems, Kunming, China. PP.115-119, October 2008.

8. Dr.E.Ramaraj, A.Senthilrajan, "Multi Core processor to support network parallel Image processing applications", International conference on signal processing system 15-17 May,2009,Singapore, Organized by International association of computer science and IT. IEEE computer society, ISBN: 978-07695-3654-5, pp.42-45.

9. Dr.E.Ramaraj, A.Senthilrajan, International multi conference of engineers and computer scientists, 17-19, March,2010, "High Density Impulse Noise removal using Median Filter", Hongkong, International Association of Engineers. ISBN: 978988-18210-4-1, ISSN: 2078-0958. Pp1481-1485.

10. Dr.E.Ramaraj, A.Senthilrajan, "Median Filter Using Multiprocessing in Agriculture", in $10^{\text {th }}$ International Conference on Signal Processing (ICSP'10), Oct.24-28, 2010, Beijing, China, Organized by IEEE Signal Processing Society. pp. 232235.

11. Dr.A.Senthilrajan,"Paddy Grade and Dirt classification using Image Processing Techniques", in C2SPCA2013Oct 10, 11, 2013, IEEE conference in Bangalore, India.

12. Dr.E.Ramaraj, A.Senthilrajan, "Median Filter in Agriculture", in World Congress on Engineering and Computer Science 2010 SanFrancisco, USA,, pp. 604-607, Organized by the International Association of Engineers (IAENG). ISBN: 978988-17012-0-6.

13. Dr.A.Senthilrajan, "Defective Chicks Deduction Based on Texture Feature Analysis Using Random Transform", in International Conference on Applied Science and Engineering held during December 05-07, 2017 at Seoul, South Korea. Presented and published.

14. Dr.A.Senthilrajan, "Comparative Study of Retrieval of Image Using Histogram and Entropy" in National Conference on organized by Skill Development Centre, Alagappa University, and Karaikudi held on $23^{\text {rd }}$ March 2015.Presented and Published.

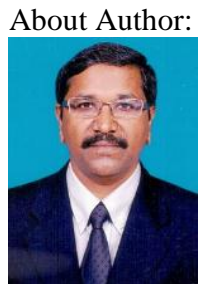

Author is working as Director in Alagappa University, Karaikudi. His area of interest in research is Digital image processing. He had teaching experience of nearly 28 years and research experience of about 10 years 